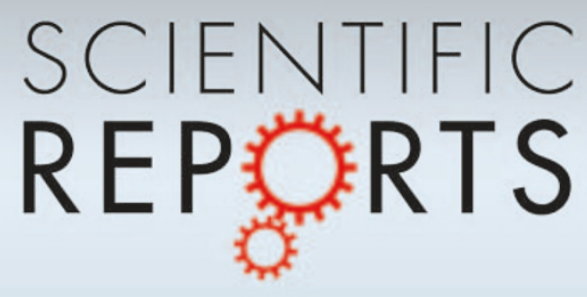

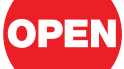

SUBJECT AREAS:

OPTICAL MATERIALS AND

DEVICES

ELECTRONIC MATERIALS AND

DEVICES

APPLIED PHYSICS

LIGHT SOURCES

Received

26 January 2012

Accepted

16 March 2012

Published

2 April 2012

Correspondence and requests for materials should be addressed to

K.T. (tanabe@iis.utokyo.ac.jp)

\section{III-V/Si hybrid photonic devices by direct fusion bonding}

\author{
Katsuaki Tanabe', Katsuyuki Watanabe' \& Yasuhiko Arakawa ${ }^{1,2}$ \\ ${ }^{1}$ Institute for Nano Quantum Information Electronics, University of Tokyo, 4-6-1 Komaba, Meguro, Tokyo 153-8505, Japan, ${ }^{2}$ Institute \\ of Industrial Science, University of Tokyo, 4-6-1 Komaba, Meguro, Tokyo 153-8505, Japan.
}

Monolithic integration of III-V compound semiconductors on silicon is highly sought after for high-speed, low-power-consumption silicon photonics and low-cost, light-weight photovoltaics. Here we present a $\mathrm{GaAs} / \mathrm{Si}$ direct fusion bonding technique to provide highly conductive and transparent heterojunctions by heterointerfacial band engineering in relation to doping concentrations. Metal- and oxide-free $\mathrm{GaAs} / \mathrm{Si}$ ohmic heterojunctions have been formed at $300^{\circ} \mathrm{C}$; sufficiently low to inhibit active material degradation. We have demonstrated $1.3 \mu \mathrm{m} \mathrm{InAs/GaAs} \mathrm{quantum} \mathrm{dot} \mathrm{lasers} \mathrm{on} \mathrm{Si} \mathrm{substrates} \mathrm{with} \mathrm{the} \mathrm{lowest} \mathrm{threshold}$ current density of any laser on Si to date, and AlGaAs/Si dual-junction solar cells, by $p$-GaAs/p-Si and $p$-GaAs/n-Si bonding, respectively. Our direct semiconductor bonding technique opens up a new pathway for realizing ultrahigh efficiency multijunction solar cells with ideal bandgap combinations that are free from lattice-match restrictions required in conventional heteroepitaxy, as well as enabling the creation of novel high performance and practical optoelectronic devices by III-V/Si hybrid integration.

II-V semiconductor compound light sources integrated onto Si chips ${ }^{1-9}$ or waveguides ${ }^{10-16}$ are promising for the realization of photonic integrated circuits ${ }^{17,18}$ utilizing well-established complementary metal-oxide-semiconductor (CMOS) fabrication technologies. Such III-V/Si hybrid devices would compensate for the poor ability of silicon to act as a light source due to its low radiative recombination rate stemming from indirect energy bandgaps. For solar cell applications, Si-based multijunction stacking would provide high efficiency ${ }^{19,20}$, low cost, mechanical robustness and light weight cells, relative to conventional $\mathrm{Si}$ and III-V multijunction cells. Additionally, heterojunction tunnel field-effect transistors consisting of low bandgap III-V semiconductors and $\mathrm{Si}$ are promising for the realization of high-density, low-power-consumption very-large-scale-integration (VLSI) by enhanced drive current relative to conventional Si-only transistors ${ }^{21,22}$. For III-V/Si hybrid integration, direct epitaxial growth of III-V compounds on Si substrates would be the most desirable approach, but heteroepitaxy typically introduces a substantial crystalline defect density due to the large lattice mismatch and the polarnonpolar nature of the III-V/IV semiconductor system that can adversely affect device performance ${ }^{23-25}$. Wafer bonding, on the other hand, is not subject to the lattice matching limitations associated with epitaxial growth, and heterostructure devices fabricated via wafer bonding can, in principle, have a performance close to those obtained by homoepitaxy by confining the defect network needed for lattice mismatch accommodation to the bonded interfaces ${ }^{26}$. It is known that ohmic $\mathrm{Si} / \mathrm{Si}$ junctions of the same polarity, i.e. $p$ - $\mathrm{Si} / p$-Si and $n$-Si $/ n$-Si, can be relatively easily obtained by direct wafer bonding even at room temperature ${ }^{26,27}$. It is, however, not the case for compound semiconductors such as GaAs and InP, and ohmic $p$-type/p-type or $n$-type/ $n$-type junction formation has required high temperature bonding above $600^{\circ} \mathrm{C}^{28-33}$ which would severely degrade the device materials. For GaAs/Si bonding that would be particularly attractive for fabrication of high performance photonic and photovoltaic devices, no successfully direct-bonded ohmic junctions have been reported, and bonding even at $700^{\circ} \mathrm{C}$ was reported to have failed ${ }^{27,34}$. Although an alternative method to prepare ohmic heterointerfaces uses metal bonding agents $s^{5,35,36}$, the bonding metal layers would shadow light as well as cause photon absorption loss and, therefore, is not ideal for optoelectronic applications. In this work, we have succeeded in preparing ohmic $\mathrm{GaAs} / \mathrm{Si}$ heterojunctions, to realize both optical transparency and electrical conductivity, by direct bonding at $300^{\circ} \mathrm{C}$ in ambient air for $p$-GaAs/p-Si homopolarity-junctions and $p$-GaAs $/ n$-Si tunnel-junctions by applying heavy, degenerating doping at the GaAs and Si surfaces to be bonded to enhance the GaAs/Si interfacial conductivity.

\section{Results}

GaAs/Si direct fusion bonding. We investigated experimentally the bonding of GaAs and Si wafers with varying doping concentrations and bonding temperature, and we characterized the GaAs/Si heterointerfacial electrical conductivities. The doping concentrations of the $p$-GaAs wafers, $p^{+}$-GaAs layers, $p^{+}-\mathrm{Si}$, and $n^{+}-\mathrm{Si}$ wafers were 
$9 \times 10^{18} \mathrm{~cm}^{-3} \mathrm{Zn}, 5 \times 10^{19} \mathrm{~cm}^{-3} \mathrm{Zn}, 3 \times 10^{19} \mathrm{~cm}^{-3} \mathrm{~B}$, and $3 \times 10^{19} \mathrm{~cm}^{-3}$ As, respectively. Current-voltage $(I-V)$ curves, under the measurement configuration shown schematically in Fig. 1a, for the bonded GaAs/Si wafer pairs are shown in Fig. $1 \mathrm{~b}$ and 1c. Rectified,

a
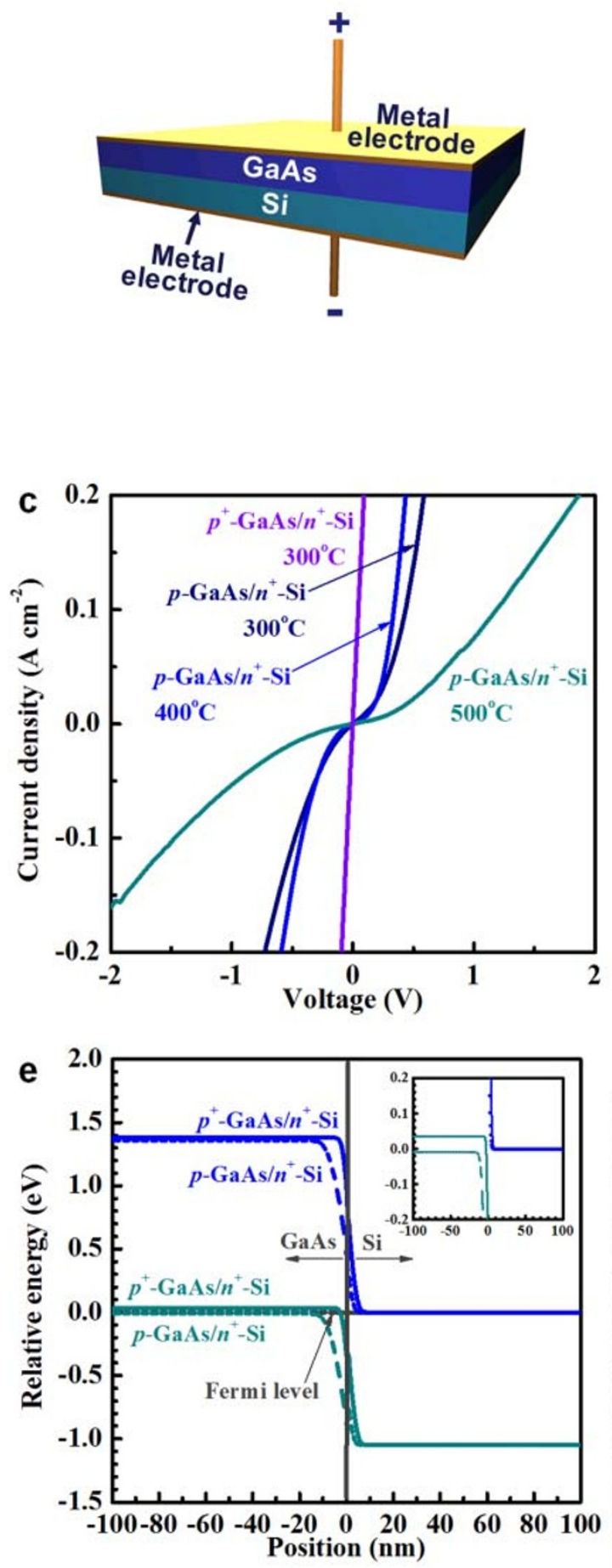

non-ohmic behaviour is seen for all $p$-GaAs $/ p^{+}-\mathrm{Si}$ and $p-\mathrm{GaAs} / n^{+}-\mathrm{Si}$ pairs including those annealed at $500^{\circ} \mathrm{C}$. In contrast to the non-ohmic $I-V$ characteristics for the $p-\mathrm{GaAs} / p^{+}-\mathrm{Si}$ and $p-\mathrm{GaAs} / n^{+}-\mathrm{Si}$ pairs, the $p^{+}-\mathrm{GaAs} / p^{+}-\mathrm{Si}$ and $p^{+}-\mathrm{GaAs} / n^{+}-\mathrm{Si}$ pairs exhibit ohmic $I-V$ curves as
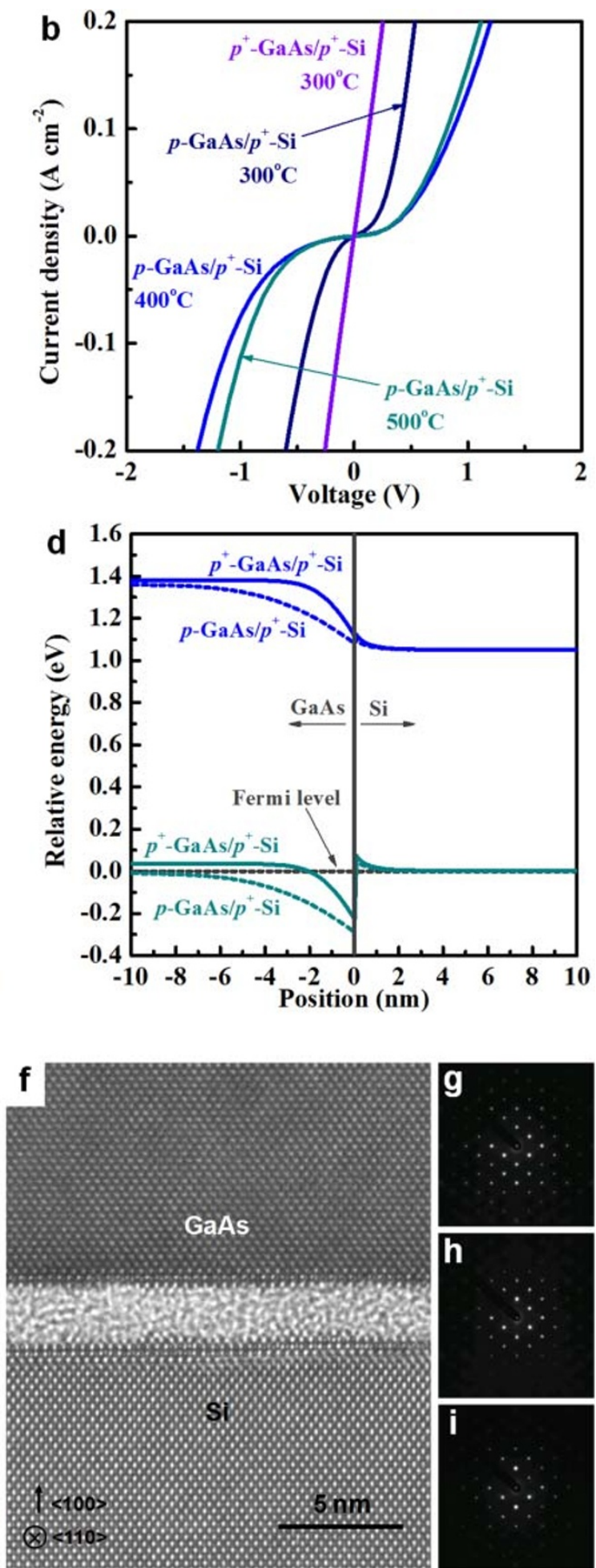

h

Figure $1 \mid \mathrm{GaAs} / \mathrm{Si}$ direct wafer bonding. (a) Configuration schematic of the $I-V$ measurement for the direct-bonded GaAs/Si heterointerfacial electrical characteristics. A positive bias voltage was applied from the GaAs side. (b, c) $I-V$ characteristics of the direct-bonded GaAs/Si heterointerfaces with varying doping concentrations and bonding temperature. (d, e) Calculated profiles of the conduction and valence band edges across the $(\mathrm{d}) p$-GaAs/ $p$-Si and (e) $p$-GaAs $/ n$-Si heterointerfaces with varying doping concentrations. The inset in e shows a closeup around the origin. (f) Cross-sectional transmission electron microscope image of a direct-bonded $p^{+}-\mathrm{GaAs} / p^{+}-\mathrm{Si}$ heterointerface. (g-i) Selected-area diffraction patterns at the same heterointerface as of $\mathbf{f}$ for regions around $70 \mathrm{~nm}$ in radius centred $(\mathrm{g}) 80 \mathrm{~nm}$ above the interface, (h) at the interface, and (i) $80 \mathrm{~nm}$ below the interface, identified as single-crystal GaAs, a mixture of single-crystal GaAs and $\mathrm{Si}$, and single-crystal Si, respectively. 
seen in Fig. $1 \mathrm{~b}$ and 1c. A cross-sectional transmission electron microscope image at a direct-bonded $p^{+}-\mathrm{GaAs} / p^{+}$Si heterointerface is shown in Fig. 1f. An amorphous layer at the GaAs/Si interface with a thickness of around $2 \mathrm{~nm}$ can be seen in the image. Even if this interfacial layer is an oxide, this thickness is sufficiently thin to provide ohmic interfacial conductivity by inducing a tunnelling current or by oxide breakdown by the applied voltage $^{37,38}$. Selected-area diffraction patterns at and around the GaAs/Si heterointerface, shown in Fig. $1 \mathrm{~g}-\mathrm{i}$, verify that the regions immediately above and below the amorphous layer are singlecrystalline $\mathrm{GaAs}$ and $\mathrm{Si}$, respectively. The images indicate that both the GaAs and Si materials remain single crystals during our bonding process with no threading dislocation generation observed around the vicinity of the bonded heterointerface. This is in contrast to interfaces in the cases of lattice-mismatched heteroepitaxy ${ }^{23-25}$.

III-V quantum dot lasers on Si substrates. As a demonstration of our GaAs/Si direct bonding technique applied to optoelectronic devices, we have fabricated semiconductor lasers using selfassembled InAs quantum dots embedded in GaAs (InAs/GaAs quantum dot lasers ${ }^{39}$ ) on $\mathrm{Si}$ substrates and operated by current injection through direct-bonded $\mathrm{GaAs} / \mathrm{Si}$ heterointerfaces. A double-hetero InAs/GaAs quantum dot laser structure was grown on a GaAs substrate by molecular beam epitaxy and layer-transferred onto a $p^{+}-\mathrm{Si}$ substrate by means of $p^{+}-\mathrm{GaAs} / p^{+}$-Si direct bonding at $300^{\circ} \mathrm{C}$ and subsequent removal of the GaAs substrate. The finished device consists of a 3.9- $\mu$ m-thick III-V semiconductor double-hetero laser structure on top of a Si substrate, as shown in Fig. 2a and 2b. Fig. $2 \mathrm{c}$ shows the light-current characteristics of the fabricated device under $500 \mathrm{~Hz}, 400 \mathrm{~ns}$ pulsed pumping at room temperature. The clear kink in the light-current curve indicates the lasing turn-on with a threshold current density of $205 \mathrm{~A} \mathrm{~cm}^{-2}$; the lowest threshold current density, to the best of our knowledge, of any kind of laser on Si. The inset of Fig. $2 c$ shows the $I-V$ characteristics of the laser. The resistivity in the linear $I-V$ region at higher voltages is around 0.1 $\Omega \mathrm{cm}^{2}$, which is the same order of magnitude as the bonded $p^{+}-\mathrm{GaAs} /$ $p^{+}$-Si bare wafer heterointerface shown in Fig. 1b. Fig. $2 d$ and $2 \mathrm{e}$ show the electroluminescence spectra at current densities of 140 and $380 \mathrm{~A} \mathrm{~cm}^{-2}$, corresponding to spontaneous and lasing emission, respectively. Room temperature lasing at the $1.3 \mu \mathrm{m}$ optical communication band, associated with the ground state transition of the InAs quantum dots, is observed. Additionally, an onset of room temperature continuous-wave lasing has been observed in a same type of sample (see Supplementary Information). a

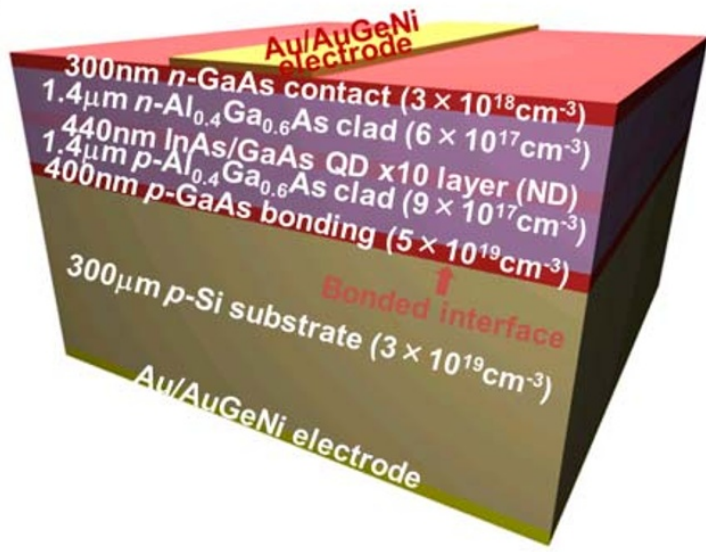

b
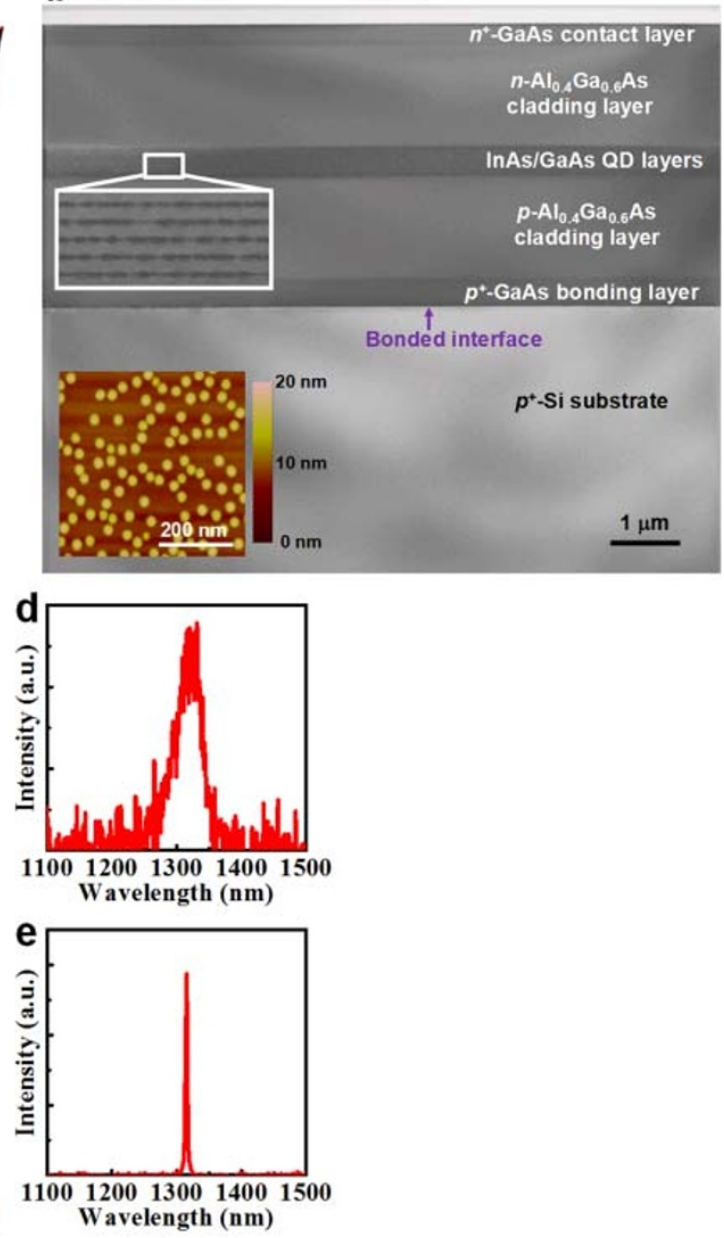

Figure $2 \mid$ InAs/GaAs quantum dot laser on Si substrate. (a) Cross-sectional schematic diagram of the fabricated InAs/GaAs quantum dot laser on a Si substrate. The thickness and doping concentration of each layer are indicated. The abbreviations QD and ND stand for quantum dot and non-doped, respectively. (b) Cross-sectional transmission electron microscope image of the laser. The upper inset shows a detailed image of the InAs/GaAs quantum dot layers. The lower inset shows an atomic force microscope image of the as-grown InAs/GaAs quantum dots. (c) Light-current characteristics of the laser for pulsed electrical pumping at room temperature. The $I-V$ characteristics of the laser are shown in the inset. (d, e) Electroluminescence spectra of the laser at current densities of 140 (below the lasing threshold) and 380 (above the lasing threshold) $\mathrm{A} \mathrm{cm}^{-2}$, respectively. 
III-V/Si multijunction solar cells. We have also fabricated AlGaAs/ $\mathrm{Si}$ dual-junction solar cells using the direct bonding technique. An $\mathrm{Al}_{0.1} \mathrm{Ga}_{0.9} \mathrm{As}$ subcell was grown on a $\mathrm{GaAs}$ substrate by molecular beam epitaxy and layer-transferred onto a Si subcell by means of the $p^{+}-\mathrm{GaAs} / n^{+}$-Si direct bonding at $300^{\circ} \mathrm{C}$ and subsequent removal of the GaAs substrate. Fig. $3 \mathrm{a}$ and $3 \mathrm{~b}$ show a cross-sectional schematic diagram and scanning electron microscope image of the fabricated $\mathrm{AlGaAs} / \mathrm{Si}$ dual-junction solar cell, respectively. Both the $\mathrm{Al}_{0.1} \mathrm{Ga}_{0.9} \mathrm{As}$ and Si subcells had $n$-on- $p$ structures, and the bonding of the $p^{+}$$\mathrm{GaAs} / n^{+}$-Si heterointerface acts as a tunnel junction to switch the polarity. The light $I-V$ and power-voltage characteristics of the solar cell under a $600 \mathrm{~nm}$-peaked halogen white light source of a one-sun intensity $\left(100 \mathrm{~mW} \mathrm{~cm}^{-2}\right)$ are shown in the inset of Fig. 3b. The device performance parameters for this solar cell are $J_{s c}=27.9 \mathrm{~mA} \mathrm{~cm}{ }^{-2}$, $V_{o c}=1.55 \mathrm{~V}, F F=0.58$, and $\eta=25.2 \%$, where $J_{s c}, V_{o c}, F F$ and $\eta$ are the short-circuit current, open-circuit voltage, fill factor and energy conversion efficiency, respectively.

\section{Discussion}

The electrical conductivity dependence on bonding temperature seen in Fig. $1 \mathrm{~b}$ and $1 \mathrm{c}$ does not show monotonic behavior, attributed to the trade-off between conductivity increase and decrease by formation of covalent bonds and thermal expansion mismatch between $\mathrm{GaAs}$ and $\mathrm{Si}$ at higher temperature, respectively. Interfacial oxide formation might also be a cause of higher interfacial resistivity at higher temperature for our wafer bonding process in ambient air. It should be also noted that the wafer bonding process basically contains some randomness in reproducibility for the bonded interfacial properties degradable for example even by a single particle accidental incorporation into the interface. The conductivity enhancement seen in Fig. $1 \mathrm{~b}$ and $1 \mathrm{c}$ can be explained through an analysis of the heterojunction band offset at the GaAs/Si interfaces. One-dimensional simulations of the heterojunction bandbending (PC1D software, University of New South Wales) indicate thinning of the potential barrier at the valence band edge due to the change of the doping concentration in GaAs from $9 \times 10^{18} \mathrm{~cm}^{-3}$ to $5 \times 10^{19} \mathrm{~cm}^{-3}$ for the $p$-type/ $p$-type pairs as seen in Fig. 1d, leading to the interfacial electrical conductivity enhancement. On the other hand, simulations indicate tunnel junction formation due to the same change in doping concentration in GaAs for the $p$-type/ $n$-type pairs as seen in Fig. 1e. This valence-band-edge rising on the GaAs side enables tunnelling carrier transport, leading to higher conductivity and ohmic characteristics across the heterojunction interfaces. These $p$-type/ $p$-type and $p$-type/ $n$-type $\mathrm{GaAs} / \mathrm{Si}$ ohmic heterojunctions are very suitable for next-generation III-V/Si hybrid optoelectronic devices that will enable both optical and electrical interconnections.

We have fabricated hundreds of lasers in a single wafer bonding step demonstrating the advantage of this approach for high volume, low cost integration over the conventional pick-and-place scheme $e^{18,40}$. Evanescent optical coupling to underneath waveguides to fabricate so-called hybrid Si lasers ${ }^{10-16,41}$ could be realized by preparing rib structures on commercially available silicon-on-insulator substrates in advance of wafer bonding. In contrast to oxide-mediated bonding used for hybrid laser fabrication to date $\mathrm{e}^{10-15}$, conductive wafer-bonded heterointerfaces enable vertical carrier injection that prevents current spreading towards cavity stripe edges. Therefore, direct-bonded hybrid lasers have the advantages of higher quantum efficiencies and simpler fabrication without mesa etching or ion implantation for carrier confinement that was required in the fabrication of earlier lateral-current-injection III-V/Si hybrid lasers ${ }^{41}$.

The low FF seen in Fig. $3 b$ is likely due to the large series resistance. However, the wafer-bonded GaAs/Si heterojunction interfacial resistance with exactly same doping concentrations in GaAs and $\mathrm{Si}$ to those used for the bonding surfaces in the dual-junction solar cell seen in Fig. 1c is far lower than the total series resistance of the dualjunction solar cell estimated from the light $I-V$ characteristics. We therefore attribute the low $F F$ principally to insufficient optimization of our front metal contact grids. Very high efficiency, over $30 \%$ under 1 sun, seems quite realistic simply through a contact redesign and would be expected based on the $J_{s c}$ and $V_{o c}$ values obtained at this preliminary research stage. To the best of our knowledge, while there have been two reports for all-III-V bonded multijunction solar cells ${ }^{42,43}$, this is the first bonded multijunction solar cell with a Si subcell. Our monolithic AlGaAs/Si dual-junction solar cell (overcoming a 4\% lattice-mismatch between $\mathrm{AlGaAs}$ and $\mathrm{Si}$ ) has demonstrated a proof-of-principle for the viability of direct wafer bonding for solar cell applications. This wafer-bonding interconnecting approach is extendable to ultrahigh efficiency multijunction solar cells, such as InGaN/AlGaAs/Si/Ge four-junction solar cells, with optimal subcell bandgap sequences free from the lattice-matching restriction required in conventional heteroepitaxy. In this work, we adopted an etch-back method to detach the GaAs growth substrate to simplify the fabrication process. Alternatively, the incorporation of an epitaxial lift-off ${ }^{6,44,45}$ or ion-cutting ${ }^{46-48}$ technique would enable the reuse of the GaAs substrates to reduce the production costs.

In conclusion, we have investigated $\mathrm{GaAs} / \mathrm{Si}$ direct wafer bonding for electrically conductive, optically transparent materials interconnection in conjunction with heterointerfacial energy band alignment calculations in relation to doping concentrations. Heavy, degenerating doping at the GaAs and Si surfaces to be bonded is found to be
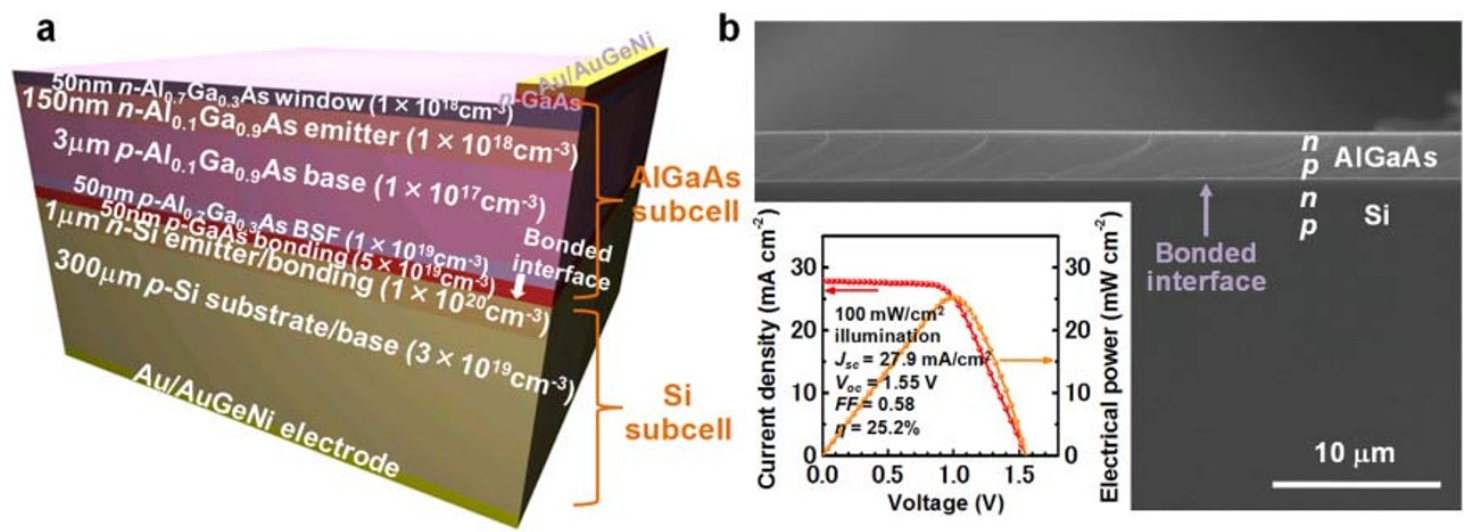

Figure $3 \mid \mathrm{AlGaAs} /$ Si dual-junction solar cell. (a) Cross-sectional schematic diagram of the fabricated AlGaAs/Si dual-junction solar cell. The thickness and doping concentration of each layer are indicated. The abbreviation BSF stands for back surface field. (b) Cross-sectional scanning electron microscope image of the solar cell. Inset shows the light $I-V$ and power-voltage characteristics of the solar cell under a $600 \mathrm{~nm}$-peaked halogen white light source of a one-sun intensity $\left(100 \mathrm{~mW} \mathrm{~cm}^{-2}\right)$. 
significant for enhancing the GaAs/Si interfacial conductivity and results in ohmic GaAs/Si heterointerfaces even for bonding temperatures of as low as $300^{\circ} \mathrm{C}$ for both $p$-type/p-type and $p$-type/ $n$-type combinations. Utilizing the $p^{+}-\mathrm{GaAs} / p^{+}-\mathrm{Si}$ and $p^{+}-\mathrm{GaAs} / n^{+}-\mathrm{Si}$ direct bonding, we have demonstrated a low threshold III-V laser on a $\mathrm{Si}$ substrate and a high-efficiency III-V/Si multijunction solar cell, respectively. Our low-temperature direct semiconductor bonding technique opens up a new pathway for realizing highperformance III-V/Si hybrid optoelectronics.

\section{Methods}

Wafer bonding and layer transfer. Bonding surfaces of the GaAs (100) and $\mathrm{Si}(100)$ wafers were first coated with a photoresist to protect the bonding surfaces from particles generated in the dicing process. The wafers were then diced into $\sim 1 \mathrm{~cm}^{2}$ area dies. The photoresist was then removed with acetone, and the bonding surfaces were degreased. Native oxide was removed by dipping both the GaAs and Si wafer pieces in HF aq. ( $20 \mathrm{vol} \%$ ) for $30 \mathrm{~s}$. The two die pieces were then brought into contact with the (011) edges aligned, which is useful for cleavage in the laser fabrication, and annealed at $300-500^{\circ} \mathrm{C}$ in ambient air for $3 \mathrm{~h}$ under a uniaxial pressure of $0.1 \mathrm{MPa}$. The bonded GaAs/Si interfacial $I-V$ characteristics were measured by a DC bias source with $\mathrm{AuGeNi} / \mathrm{Au}$ ohmic contacts applied to the outer surfaces of the bonded $\mathrm{GaAs} / \mathrm{Si}$ pieces. Bonded pairs with varying doping concentrations at the subsequent bond interfaces were investigated. Highly doped $p^{+}$-GaAs layers were prepared by metallorganic chemical vapor deposition on the $p$-GaAs (100) wafers. The bonding yields in relation to surface roughness appear in Supplementary Information.

The laser and solar cell structures were layer transferred onto a $p^{+}-\mathrm{Si}(100)$ substrate and Si solar cell wafer (details described in Multijunction solar cells section), respectively, through wafer bonding and subsequent removal of the GaAs substrate. After direct semiconductor wafer bonding at $300^{\circ} \mathrm{C}$, the GaAs substrate was removed at room temperature by selective chemical etching with $\mathrm{H}_{3} \mathrm{PO}_{4}-\mathrm{H}_{2} \mathrm{O}_{2}(3: 7$ vol.) followed by $50 \%$ citric acid- $\mathrm{H}_{2} \mathrm{O}_{2}(4: 1 \mathrm{vol}$.), with the edges of the $\mathrm{GaAs}$ wafer coated with photoresist to avoid undercutting of the quantum dot laser structure. The $\mathrm{H}_{3} \mathrm{PO}_{4}-\mathrm{H}_{2} \mathrm{O}_{2}$ and citric acid- $\mathrm{H}_{2} \mathrm{O}_{2}$ solution compositions were chosen to maximize the etching rate of GaAs and the etching selectivity between GaAs and AlGaAs, respectively ${ }^{4}$. The $\mathrm{Al}_{0.7} \mathrm{Ga}_{0.3}$ As etch stop layer was then removed by $\mathrm{HCl}$ aq. (conc.) or $\mathrm{HF}$ aq. $(20 \mathrm{vol} \%)$ at room temperature.

Quantum dot lasers. The InAs/GaAs quantum dot laser structure was grown on GaAs (100) substrate by molecular beam epitaxy. The laser structure consisted of a 440nm-thick GaAs layer embedded with ten layers of self-assembled InAs quantum dots with a density per layer of $3.8 \times 10^{10} \mathrm{~cm}^{-2}$. The GaAs layer was clad with $1.4-\mu \mathrm{m}$-thick $\mathrm{Al}_{0.4} \mathrm{Ga}_{0.6} \mathrm{As}$. An $\mathrm{Al}_{0.7} \mathrm{Ga}_{0.3}$ As etch stop layer was grown between the GaAs substrate and the lower $\mathrm{Al}_{0.4} \mathrm{Ga}_{0.6}$ As clad. The as-grown InAs quantum dots exhibited a photoluminescence peak associated with the ground state emission at $1.3 \mu \mathrm{m}$ with a full width at half maximum of $30 \mathrm{meV}$ at room temperature. Following the wafer bonding and layer transfer described above, broad-area Fabry-Perot lasers with cleaved facets were formed by applying $\mathrm{Au} / \mathrm{AuGeNi}$ electrodes to the top and bottom of the structure. The finished device is a 3.9- $\mu \mathrm{m}$-thick double-hetero laser structure bonded to a $\mathrm{Si}$ substrate with no mediating agent. The laser cavity length and width were $2.1 \mathrm{~mm}$ and $100 \mu \mathrm{m}$, respectively. A high-reflection coating was not applied to the cleaved edges.

Multijunction solar cells. The $\mathrm{Al}_{0.1} \mathrm{Ga}_{0.9} \mathrm{As}$ subcell, with a bandgap energy of $1.6 \mathrm{eV}$, was a $p-n \mathrm{Al}_{0.1} \mathrm{Ga}_{0.9} \mathrm{As} / \mathrm{Al}_{0.7} \mathrm{Ga}_{0.3}$ As double-hetero structure. The subcell was grown inversely as $p$-on- $n$ on a GaAs (100) substrate by molecular beam epitaxy with an $\mathrm{Al}_{0.7} \mathrm{Ga}_{0.3} \mathrm{As}$ etch stop layer immediately above the $\mathrm{GaAs}$ substrate. This results in an $n$-on- $p$ top subcell in the final bonded dual-junction cell structure. The Si subcell, with a bandgap energy of $1.1 \mathrm{eV}$, was prepared by thermal diffusion of $\mathrm{P}$ from phosphoricacid-based glass into the surface region of a $p$-type $\mathrm{Si}(100)$ wafer. Specifically, the $\mathrm{GaAs}$ subcell was terminated with a Be-doped GaAs layer with a carrier concentration of $5 \times 10^{19} \mathrm{~cm}^{-3}$, and the Si subcell was terminated with a P-doped Si layer with a carrier concentration of $1 \times 10^{20} \mathrm{~cm}^{-3}$. These subcells were directly bonded and followed by $\mathrm{GaAs}$ substrate removal, metallization with $\mathrm{Au} / \mathrm{AuGeNi}$, and application of an antireflection coating with $\mathrm{MgF}_{2} / \mathrm{ZnS}$

1. Seassal, C. et al. InP microdisk lasers on silicon wafer: $\mathrm{CW}$ room temperature operation at $1.6 \mu \mathrm{m}$. Electron. Lett. 37, 222-223 (2001).

2. Monat, C. et al. InP-based two-dimensional photonic crystal on silicon: In-plane Bloch mode laser. Appl. Phys. Lett. 81, 5102-5104 (2002).

3. Palit, S. et al. Low-threshold thin-film III-V lasers bonded to silicon with front and back side defined features. Opt. Lett. 34, 2802-2804 (2009).

4. Tanabe, K., Nomura, M., Guimard, D., Iwamoto, S. \& Arakawa, Y. Room temperature continuous wave operation of InAs/GaAs quantum dot photonic crystal nanocavity laser on silicon substrate. Opt. Express 17, 7036-7042 (2009).

5. Tanabe, K., Guimard, D., Bordel, D., Iwamoto, S. \& Arakawa, Y. Electrically pumped $1.3 \mu \mathrm{m}$ room-temperature InAs/GaAs quantum dot lasers on $\mathrm{Si}$ substrates by metal-mediated wafer bonding and layer transfer. Opt. Express 18, 10604-10608 (2010).
6. Yoon, J. et al. GaAs photovoltaics and optoelectronics using releasable multilayer epitaxial assemblies. Nature 465, 329-334 (2010).

7. Kim, R. et al. Waterproof AlInGaP optoelectronics on stretchable substrates with applications in biomedicine and robotics. Nature Mater. 9, 929-937 (2010).

8. Ko, H. et al. Ultrathin compound semiconductor on insulator layers for highperformance nanoscale transistors. Nature 468, 286-289 (2010).

9. Chen, R. et al. Nanolasers grown on silicon. Nature Photon. 5, 170-175 (2011).

10. Fang, A. W. et al. Electrically pumped hybrid AlGaInAs-silicon evanescent laser. Opt. Express 14, 9203-9210 (2006).

11. Van Campenhout, J. et al. Electrically pumped InP-based microdisk lasers integrated with a nanophotonic silicon-on-insulator waveguide circuit. Opt Express 15, 6744-6749 (2007).

12. Roelkens, G. et al. III-V/Si photonics by die-to-wafer bonding. Mater. Today 10, (7-8) 36-43 (2007).

13. Sun, X. et al. Electrically pumped hybrid evanescent $\mathrm{Si} / \mathrm{InGaAsP}$ lasers. Opt. Lett. 34, 1345-1347 (2009).

14. Roelkens, G. et al. III-V/silicon photonics for on-chip and inter-chip optical interconnects. Laser Photon. Rev. 4, 751-779 (2010).

15. Liang, D. \& Bowers, J. E. Recent progress in lasers on silicon. Nature Photon. 4, 511-517 (2010).

16. Halioua, Y. et al. Hybrid III-V/Si semiconductor/silicon nanolaser. Opt. Express 19, 9221-9231 (2011).

17. Miller, D. A. B. Rationale and challenges for optical interconnects to electric chips. Proc. IEEE 88, 728-749 (2000).

18. Kato, K. \& Tohmori, Y. PLC hybrid integration technology and its application to photonic components. IEEE J. Select. Top. Quant. Electron. 6, 4-13 (2000).

19. Kurtz, S. R., Faine, P. \& Olson, J. M. Modeling of two-junction, series-connected tandem solar cells using top-cell thickness as an adjustable parameter. J. Appl. Phys. 68, 1890-1895 (1990)

20. Umeno, M., Soga, T., Baskar, K. \& Jimbo, T. Heteroepitaxial technologies on Si for high-efficiency solar cells. Sol. Ener. Mater. Sol. Cells 50, 203-212 (1998).

21. Verhulst, A. S. et al. Complementary silicon-based heterostructure tunnel-FETs with high tunnel rates. IEEE Electron. Dev. Lett. 29, 1398-1401 (2008).

22. Bjork, M. T. et al. Si-InAs heterojunction Esaki tunnel diodes with high current densities. Appl. Phys. Lett. 97, 163501 (2010).

23. Kroemer, H., Liu, T-Y. \& Petroff, P. M. GaAs on Si and related systems: Problems and prospects. J. Cryst. Growth 95, 96-102 (1989).

24. Sugo, M., Takanashi, Y., Aljassim, M. M. \& Yamaguchi, M. Heteroepitaxial growth and characterization of InP on Si substrates. J. Appl. Phys. 68, 540-547 (1990).

25. Shimizu, Y. \& Okada, Y. Growth of high-quality GaAs/Si films for use in solar cell applications. J. Cryst. Growth 265, 99-106 (2004).

26. Tong, Q. -Y. \& Gosele, U. Semiconductor wafer bonding: Science and technology (Wiley, New Jersey, 1998)

27. Howlader, M. M. R., Watanabe, T. \& Suga, T. Investigation of the bonding strength and interface current of $\mathrm{p}-\mathrm{Si} / \mathrm{n}-\mathrm{GaAs}$ wafers bonded by surface activated bonding at room temperature. J. Vac. Sci. Technol. B 19, 2114-2118 (2001).

28. Kish, F. A. et al. Low-resistance Ohmic conduction across compound semiconductor wafer-bonded interfaces. Appl. Phys. Lett. 67, 2060-2062 (1995).

29. Okuno, Y., Uomi, K., Aoki, M. \& Tsuchiya, T. Direct wafer bonding of III-V compound semiconductors for free-material and free-orientation integration. IEEE J. Quant. Electron. 33, 959-969 (1997).

30. Salomonsson, F. et al. Wafer-fused p-InP/p-GaAs heterojunctions. J. Appl. Phys. 83, 768-774 (1998)

31. Hammar, M. et al. Systematics of electrical conductivity across InP to GaAs waferfused interfaces. Jpn. J. Appl. Phys. 38, 1111-1114 (1999).

32. Kastner, G., Breitenstein, O., Scholz, R. \& Reiche, M. Compound semiconductor interfaces obtained by direct wafer bonding in hydrogen or forming gas. J. Mater. Sci. Mater. Electron. 13, 593-595 (2002)

33. Shi, F. et al. Characterization of GaAs-based n-n and p-n interface junctions prepared by direct wafer bonding. J. Appl. Phys. 92, 7544-7549 (2002).

34. Zhou, Y. C., Zhu, Z. H., Crouse, D. \& Lo, Y. H. Electrical properties of waferbonded GaAs/Si heterojunctions. Appl. Phys. Lett. 73, 2337-2339 (1998).

35. Arokiaraj, J. et al. Electrical characteristics of GaAs bonded to Si using $\mathrm{SeS}_{2}$ technique. Jpn. J. Appl. Phys. 39, L911-L913 (2000).

36. Bickford, J. R., Qiao, D., Yu, P. K. L. \& Lau, S. S. Electrical characterization of GaAs metal bonded to Si. Appl. Phys. Lett. 89, 012106 (2006).

37. Rosenbaum, E. \& Register, L. F. Mechanism of stress-induced leakage current in MOS capacitors. IEEE Trans. Electron. Dev. 44, 317-323 (1997).

38. Miranda, E., Sune, J., Rodriguez, R., Nafria, M. \& Aymerich, X. Soft breakdown fluctuation events in ultrathin $\mathrm{SiO}_{2}$ layers. Appl. Phys. Lett. 73, 490-492 (1998).

39. Arakawa, Y. \& Sakaki, H. Multidimensional quantum well laser and temperature dependence of its threshold current. Appl. Phys. Lett. 40, 939-941 (1982).

40. Friedrich, E. E. L., Oberg, M. G., Broberg, B., Nilsson, S. \& Valette, S. Hybrid integration of semiconductor lasers with Si-based single-mode ridge waveguides. J. Lightwave Technol. 10, 336-340 (1992).

41. Tanabe, K., Iwamoto, S. \& Arakawa, Y. Novel III-V/Si hybrid laser structures with current injection across conductive wafer-bonded heterointerfaces: A proposal and analysis. IEICE Electron. Express 8, 596-603 (2011).

42. Tanabe, K., Fontcuberta i Morral, A., Atwater, H. A., Aiken, D. J. \& Wanlass, M. W Direct-bonded GaAs/InGaAs tandem solar cell. Appl. Phys. Lett. 89, 102106 (2006).

43. Law, D. C. et al. Semiconductor-bonded III-V space multijunction solar cells. Proc 34th IEEE Photovoltaic Specialists Conference, Philadelphia, 002237-002240 (2009). 
44. Konagai, M., Sugimoto, M. \& Takahashi, K. High efficiency GaAs thin film solar cells by peeled film technology. J. Cryst. Growth 45, 277-280 (1978).

45. Yablonovitch, E., Gmitter, T., Harbison, J. P. \& Bhat, R. Extreme selectivity in the lift-off of epitaxial GaAs films. Appl. Phys. Lett. 51, 2222-2224 (1987).

46. Bruel, M. Silicon on insulator material technology. Electron. Lett. 31, 1201-1202 (1995).

47. Chen, P. et al. High crystalline-quality III-V layer transfer onto Si substrate. Appl. Phys. Lett. 92, 092107 (2008)

48. Chen, W. et al. Integration of thin film layers of single-crystalline InP with flexible substrates. Appl. Phys. Lett. 92, 212109 (2008).

\section{Acknowledgements}

The authors thank Denis Guimard and Masao Nishioka for growing the $p^{+}$-GaAs bonding layer. This research was supported by the JSPS through the FIRST Program and Kakenhi 23760303, MEXT Japan through the Special Coordination Funds for Promoting Science and Technology, and Intel Corporation.

\section{Author contributions}

K.T. conceived and designed the experiments. K.W. performed material growth. K.T. carried out the wafer bonding, calculations, device fabrication, and measurements. Y.A. supervised the overall project. K.T. and Y.A. composed the manuscript. All authors contributed to discussion of the results.

\section{Additional information}

Supplementary information accompanies this paper at http://www.nature.com/ scientificreports

Competing financial interests: The authors declare no competing financial interests.

License: This work is licensed under a Creative Commons

Attribution-NonCommercial-ShareAlike 3.0 Unported License. To view a copy of this license, visit http://creativecommons.org/licenses/by-nc-sa/3.0/

How to cite this article: Tanabe, K., Watanabe, K. \& Arakawa, Y. III-V/Si hybrid photonic devices by direct fusion bonding. Sci. Rep. 2, 349; DOI:10.1038/srep00349 (2012). 